$$
\text { "meszaros" — 2006/6/22 — 18:17 — page } 179 \text { — \#1 }
$$

\title{
Sequenced problems for functional equations
}

\author{
Zsolt ÁdÁm, KÁroly LaJKó, \\ Gyula Maksa and Fruzsina MÉszáros
}

Abstract. There are many possible methods to solve equations of the form

$$
H(f(x+y), f(x-y), f(x), f(y), x, y)=0 \quad(x, y \in \mathbb{R}),
$$

where $H$ is a known function and $f$ is the unknown function to be determined. Here we will create a sequence of problems for equations of type (1) (see on the next page). These sequenced problems are appropriate for the fostering of talented students on different level of mathematical education.

Key words and phrases: sequenced problems, functional equations.

ZDM Subject Classification: I70, U40.

\section{Introduction}

In the secondary mathematical education equations (or system of equations) containing one or more unknowns, numbers and/or parameters are usually considered and solved.

However, on mathematical competitions, in mathematical journals and problem books there are growing number of such equations (or system of equations) containing independent variables, known functions, unknown functions and constants. Those type of equations are called functional equations. A function (or a 
set of functions) is a (particular) solution of a functional equation (or a system of functional equations) if, and only if, it satisfies the functional equation (or the system of functional equations) on its given domain. A functional equation is solved in a given class of functions if the totality of (particular) solutions are given in that class.

There are many monographs for systematical discussion of the theory of functional equations (see e.g. [1], [2], [3], [6], [8], [9], [10], [12]). But, as far as we know, there are only a few textbook in this field (e.g. [4], [5], [7], [11]), which are suitable for the fostering of talented students on different level of mathematical education and might help the work of theachers, too. It is interesting that most of these monographs and textbooks are closely associated with Eastern-European scientists and teachers, who played an important role in the investigations of functional equations and in the discovering and collecting of the school-applications.

During the preparations of lecture notes [11], being supported by the fundamental monograph [2] of Aczél, we studied the following problem field.

There are many possible methods to solve functional equations of the form

$$
H(f(x+y), f(x-y), f(x), f(y), x, y)=0 \quad(x, y \in \mathbb{R})
$$

where $H$ is a known function and $f$ is the unknown function to be determined. Aczél in [2] investigated the following particular case of (1)

$$
f(x) f(x+y)=[f(y)]^{2}[f(x-y)]^{2} a^{y+4} \quad(x, y \in \mathbb{R}),
$$

where $a \in \mathbb{R}_{+}=\{x \in \mathbb{R}: x>0\}, a \neq 1$ is an arbitrary constant. He determined the identically zero and nowhere zero solutions of (2) in the form

$$
f(x)=0(x \in \mathbb{R}), \quad f(x)=a^{x-2}(x \in \mathbb{R}), \quad f(x)=-a^{x-2}(x \in \mathbb{R}) .
$$

But, it is easy to see, that functions

$$
f(x)=\left\{\begin{array}{ll}
a^{x-2} & \text { for } x \in \mathbb{Q} \\
0 & \text { for } x \in \mathbb{R} \backslash \mathbb{Q}
\end{array} \quad \text { or } \quad f(x)= \begin{cases}-a^{x-2} & \text { for } x \in \mathbb{Q} \\
0 & \text { for } x \in \mathbb{R} \backslash \mathbb{Q}\end{cases}\right.
$$

are also solutions of (2) (see [11]).

Our main aim in this paper is to obtain all solutions of functional equation (2). For this purpose we will create a sequence of problems for equations of type (1). 


\section{Two simple problems}

Problem 1. Prove that for all $c \in \mathbb{R}$ the function

$$
f(x)= \begin{cases}c & \text { for } x \in \mathbb{Q} \\ 0 & \text { for } x \in \mathbb{R} \backslash \mathbb{Q}\end{cases}
$$

satisfies the functional equation

$$
f(x) f(x+y)=f(y) f(x-y) \quad(x, y \in \mathbb{R}) .
$$

Here $\mathbb{Q}$ is the set of rational numbers.

Solution. In case $c=0, f(x)=0(x \in \mathbb{R})$ which indeed satisfies (5). In case $c \neq 0$, we distinguish four cases.

- If $x, y \in \mathbb{Q}$ then $x+y, x-y \in \mathbb{Q}$. These imply that $f(x)=f(y)=f(x+y)=$ $f(x-y)=c$. Thus (5) holds for all $x, y \in \mathbb{Q}$.

- If $x, y \in \mathbb{R} \backslash \mathbb{Q}$ then $f(x)=f(y)=0$. Hence, both sides of equation (5) is equal to 0 , which implies that (5) holds in this case, too.

- If $x \in \mathbb{Q}, y \in \mathbb{R} \backslash \mathbb{Q}$ then we have $x+y \in \mathbb{R} \backslash \mathbb{Q}$. Thus, we infer from $f(y)=f(x+y)=0$ that (5) holds for all $x \in \mathbb{Q}$ and $y \in \mathbb{R} \backslash \mathbb{Q}$.

- If $x \in \mathbb{R} \backslash \mathbb{Q}, y \in \mathbb{Q}$ then $x-y \in \mathbb{R} \backslash \mathbb{Q}$. Therefore $f(x)=f(x-y)=0$ immediately implies that $f$ satisfies (5) for all $x \in \mathbb{R} \backslash \mathbb{Q}$ and $y \in \mathbb{Q}$.

This completes the proof of the statement in Problem 1.

Problem 2. Prove that for all $c \in \mathbb{R}$ the function

$$
f(x)= \begin{cases}c & \text { for } x \in \mathbb{Z} \\ 0 & \text { for } x \in \mathbb{R} \backslash \mathbb{Z}\end{cases}
$$

satisfies equation (5). Here $\mathbb{Z}$ denotes the set of integers of $\mathbb{R}$.

Solution. The solution is like that of Problem 1 with the change that we take $\mathbb{Z}$ instead of $\mathbb{Q}$.

3. The general solution of functional equation (5)

Problem 3. Determine all solutions $f: \mathbb{R} \rightarrow \mathbb{R}$ of functional equation (5). 
Solution. Substitute $y=0$ in (5). Thus we get that

$$
[f(x)]^{2}=f(0) f(x) \quad(x \in \mathbb{R}) .
$$

If $f(0)=c$ then $f$ satisfies (6) if, and only if,

$$
f(x)[f(x)-c]=0 \quad(x \in \mathbb{R}) .
$$

This implies that $f(x) \in\{0, c\}$ for all $x \in \mathbb{R}$. Thus we have that the only possible solutions of (5) are of the form

$$
f(x)= \begin{cases}c & \text { for } x \in A \\ 0 & \text { for } x \in \mathbb{R} \backslash A\end{cases}
$$

for some set $A \subseteq \mathbb{R}$. We shall prove that functions of the form (7) satisfy (5) if, and only if, the set $A \subseteq \mathbb{R}$ has the following properties:

$$
x, y \in A \text { imply } x+y \in A ; \quad 0 \in A ; \quad x \in A \text { implies }-x \in A \text {. }
$$

We say, and this can be formulated in secondary school, $A \subseteq \mathbb{R}$ is a group with respect to the operation + of $\mathbb{R}$, that is, $A \subseteq \mathbb{R}$ is an additive subgroup of $\mathbb{R}$. First, we show that, if $A \subseteq \mathbb{R}$ is an arbitrary additive subgroup of $\mathbb{R}$ then functions $f: \mathbb{R} \rightarrow \mathbb{R}$ of the form (7) satisfy equation (5). To this, as at the solution of Problem 1, we distinguish four cases:

- If $x, y \in A$ then $x+y,-y \in A$ and so $x-y=x+(-y) \in A$. This and (7) imply $f(x)=f(y)=f(x+y)=f(x-y)=c$. Thus (5) holds for all $x, y \in A$.

- If $x, y \in \mathbb{R} \backslash A$ then $f(x)=f(y)=0$ immediately implies that (5) holds in this case, too.

- If $x \in A$ and $y \in \mathbb{R} \backslash A$ then we have $x+y \in \mathbb{R} \backslash A$ (on the contrary, let us suppose $x+y \in A$ then, since $-x \in A, y=(x+y)+(-y) \in A$, which contradicts $y \in \mathbb{R} \backslash A)$. Thus, we infer from $(7), f(y)=f(x+y)=0$. Therefore, both sides of (5) is equal to 0, that is, (5) holds for all $x \in A$, $y \in \mathbb{R} \backslash A$.

- If $x \in \mathbb{R} \backslash A$ and $y \in A$ then, similarly to the previous case, we get that $x-y \in \mathbb{R} \backslash A$ and then the equalities $f(x)=f(x-y)=0$ imply (5) for all $x \in \mathbb{R} \backslash A$ and $y \in A$.

On the other hand, we prove that if the function (7) satisfies functional equation (5) then $A$ is an additive subgroup of $\mathbb{R}$.

If $c=0$ then $A=\mathbb{R}$ is a group.

If $c \neq 0$ then 
- $f(0)=c \neq 0$ implies $0 \in A$.

- Suppose that $y \in A$. Substitute $x=0$ in (5) to obtain

$$
f(0) f(y)=f(y) f(-y) \quad(y \in A) .
$$

Thus, using the equalities $f(0)=f(y)=c$, we get $f(-y)=c$, which implies $-y \in A$.

- If $x, y \in A$ then, replacing $x$ by $x+y$ and $y$ by $x$ in (5), we get

$$
f(x+y) f(2 x+y)=f(x) f(y) \quad(x, y \in A) .
$$

Since $f(x)=f(y)=c \neq 0(x, y \in A)$, the right-hand side of the previous equation is different from 0 , thus $f(x+y) \neq 0$, that is, $x+y \in A$.

Thus $A$ is an additive subgroup of $\mathbb{R}$ which completes the proof of the last statement.

Summarizing, our solution implies the following result.

Theorem 1. The function $f: \mathbb{R} \rightarrow \mathbb{R}$ satisfies functional equation (5) if, and only if,

$$
f(x)= \begin{cases}c & \text { for } x \in A \\ 0 & \text { for } x \in \mathbb{R} \backslash A\end{cases}
$$

where $c \in \mathbb{R}$ is an arbitrary constant and $A \subseteq \mathbb{R}$ is the empty set or an arbitrary subgroup of the additive group of the set of real numbers.

Remark 1. If $A=\mathbb{Q}$ or $A=\mathbb{Z}$ then Theorem 1 implies the solutions of Problem 1 and Problem 2, respectively.

Remark 2. One can see easily the following: if there exists a point $x_{0} \in A$ such that $f$ is continuous in $x_{0}$ then the group $A$ contains a nonempty open interval. Therefore $A=\mathbb{R}$. Thus the function $f(x)=c(x \in \mathbb{R})$, where $c \in \mathbb{R}$ is an arbitrary constant, is the only continuous solution of (5).

REMARK 3. If $A \neq \mathbb{R}$ is an additive subgroup of $\mathbb{R}$ then there exist (at least at the points of $A$ ) discontinuous solutions of (5). If $A=\mathbb{Q}$ and $0 \neq c \in \mathbb{R}$ then

$$
f(x)= \begin{cases}c & \text { for } x \in \mathbb{Q} \\ 0 & \text { for } x \in \mathbb{R} \backslash \mathbb{Q}\end{cases}
$$

is a nowhere continuous solution of (5). If $c=1$ then we get the Dirichlet function as a solution of functional equation (5). 


\section{A harder problem}

Problem 4. Find all solutions $f: \mathbb{R} \rightarrow \mathbb{R}$ of the functional equation

$$
f(x) f(x+y)=[f(y)]^{2}[f(x-y)]^{2} \quad(x, y \in \mathbb{R}) .
$$

((8) is of type (1), too.)

Solution. Substitute $x=y=0$ in (8) to obtain the equality $[f(0)]^{2}=$ $[f(0)]^{4}$, which is equivalent to the equality $[f(0)]^{2}\left([f(0)]^{2}-1\right)=0$. This implies that either $f(0)=0$ or $f(0)=1$ or $f(0)=-1$.

Put $y=0$ into (8) to get

$$
[f(x)]^{2}=[f(0)]^{2}[f(x)]^{2} \quad(x \in \mathbb{R}) .
$$

In case $f(0)=0(9)$ implies $f(x)=0(x \in \mathbb{R})$, which indeed satisfies functional equation (8).

If $f(0)=1$ or $f(0)=-1$ then there is an other method for obtaining solutions of equation (8) consist of substituting $x=0$ and $x=0, y=-y$, respectively. Thus we get

$$
\begin{aligned}
f(0) f(y) & =[f(y)]^{2}[f(-y)]^{2} \\
f(0) f(-y) & =[f(-y)]^{2}[f(y)]^{2}
\end{aligned}
$$

for all $y \in \mathbb{R}$. If $(10)$ can be solved for $f(y)$, after eliminating $f(-y)$, then the function obtained from it, is the only possible solution of (8) in case $f(0)= \pm 1$. (10) shows that $f(-y)=f(y)(y \in \mathbb{R})$, that is, $f$ is an even function. Thus we get that $f$ satisfies

$$
f(0) f(y)=[f(y)]^{4} \quad(y \in \mathbb{R})
$$

or

$$
f(y)\left([f(y)]^{3}-f(0)\right)=0 \quad(y \in \mathbb{R}) .
$$

This implies that $f(y) \in\{0, \sqrt[3]{f(0)}\}$ for all $y \in \mathbb{R}$. Thus, in case $f(0)=1$ or $f(0)=-1$ the functions

$$
f(x)=\left\{\begin{array}{ll}
1 & \text { for } x \in A \\
0 & \text { for } x \in \mathbb{R} \backslash A
\end{array} \text { and } \quad f(x)= \begin{cases}-1 & \text { for } x \in A \\
0 & \text { for } x \in \mathbb{R} \backslash A,\end{cases}\right.
$$

respectively, are the only possible solutions of equation (8) for some sets $A \subseteq \mathbb{R}$. We will prove that functions of the form (11) satisfy (8) if, and only if, $A \subseteq \mathbb{R}$ is an arbitrary additive subgroup of $\mathbb{R}$. 
First, using the same argument as at the solution of Problem 3, we prove that functions of the form (11) satisfy (8) if $A$ is an additive subgroup of $\mathbb{R}$ :

- If $x, y \in A$ then $x+y, x-y \in A$. Thus $f(x)=f(y)=f(x+y)=f(x-y)= \pm 1$. Therefore (8) holds for all $x, y \in A$.

- If $x, y \in \mathbb{R} \backslash A$ then $f(x)=f(y)=0$. Hence, both sides of equation (8) is equal to 0 , which implies that (8) holds in this case, too.

- If $x \in A, y \in \mathbb{R} \backslash A$ then $x+y \in \mathbb{R} \backslash A$. Thus, we infer from $f(y)=f(x+y)=0$ that (8) holds for all $x \in A$ and $y \in \mathbb{R} \backslash A$.

- If $x \in \mathbb{R} \backslash A, y \in A$ then $x-y \in \mathbb{R} \backslash A$. Therefore $f(x)=f(x-y)=0$ immediately implies that $f$ satisfies (8) for all $x \in \mathbb{R} \backslash A$ and $y \in A$.

It remains to show that, if the functions in (11) satisfy (8) for some $A \subseteq \mathbb{R}$, then $A$ is an additive subgroup of $\mathbb{R}$.

- $f(0)= \pm 1$ implies $0 \in A$;

- If $y \in A$ then, from the first equation of system (10), we get

$$
( \pm 1)( \pm 1)=( \pm 1)^{2}[f(-y)]^{2} \quad(y \in \mathbb{R})
$$

which implies $f(-y)= \pm 1$, that is, $-y \in A$;

- If $x, y \in A$ then replacing $x$ by $x+y$ and $y$ by $x$ in (8), we get

$$
f(x+y) f(2 x+y)=[f(x)]^{2}[f(y)]^{2} \quad(x, y \in A),
$$

which implies

$$
f(x+y) f(2 x+y)=1 \quad(x, y \in A) .
$$

Thus $f(x+y) \neq 0$, that is, $x+y \notin \mathbb{R} \backslash A \Rightarrow x+y \in A$.

Thus we have proved the following result.

THEOREM 2. The function $f: \mathbb{R} \rightarrow \mathbb{R}$ satisfies functional equation (8) if, and only if, either

$$
\begin{gathered}
f(x)=0 \text { for } x \in \mathbb{R} \text { or } \\
f(x)=\left\{\begin{array}{ll}
1 & \text { for } x \in A \\
0 & \text { for } x \in \mathbb{R} \backslash A
\end{array} \quad \text { or } \quad f(x)= \begin{cases}-1 & \text { for } x \in A \\
0 & \text { for } x \in \mathbb{R} \backslash A\end{cases} \right.
\end{gathered}
$$

where $A \subseteq \mathbb{R}$ is an additive subgroup of $\mathbb{R}$.

Remark 4. Similarly to Remark 2, we get that either $f(x)=0(x \in \mathbb{R})$ or $f(x)=1(x \in \mathbb{R})$ or $f(x)=-1(x \in \mathbb{R})$ are the only continuous solutions of equation (8). 
REMARK 5. If $A$ is a proper additive subgroup of $\mathbb{R}$, then the not identically zero solutions of (8) are discontinuous at least at every point of $A$. If $A=\mathbb{Q}$, then these solutions are nowhere continuous.

REMARK 6. The last part of the proof of Problem 4 (as well as Problem 3) can be simplified by the following characterization of subgroups:

$\emptyset \neq A \subset \mathbb{R}$ is an additive subgroup of $\mathbb{R}$ if, and only if, $x-y \in A$ for every $x, y \in A$. Thus if $x, y \in A$, replacing $x$ by $y$ and $y$ by $x-y$ in (8), we get

$$
f(y) f(x)=[f(x-y)]^{2}[f(2 y-x)]^{2} \quad(x, y \in A),
$$

which immediately implies that $f(x-y) \neq 0$ and so $x-y \in A$.

\section{The general solution of equation (12)}

The next step in creating our sequenced problems is the following.

Problem 5. Determine all solutions $f: \mathbb{R} \rightarrow \mathbb{R}$ of the functional equation

$$
f(x) f(x+y)=[f(y)]^{2}[f(x-y)]^{2} a^{y+4}
$$

where $1 \neq a \in \mathbb{R}_{+}$is an arbitrary constant.

(If $a=1$ then we derive equation (8) of Problem 4 from (12).)

Solution. Set $x=y=0$ in (12) to get the equality $[f(0)]^{2}=[f(0)]^{4} a^{4}$, which can be written in the form

$$
[f(0)]^{2}\left(1-[f(0)]^{2} a^{4}\right)=0 .
$$

This implies that either $f(0)=0$ or $f(0)=\frac{1}{a^{2}}$ or $f(0)=-\frac{1}{a^{2}}$. Put $y=0$ into (12) to get

$$
[f(x)]^{2}=[f(0)]^{2}[f(x)]^{2} a^{4} \quad(x \in \mathbb{R}),
$$

which, together with $f(0)=0$, implies that $f(x)=0(x \in \mathbb{R})$.

Let now $f(0)=\frac{1}{a^{2}}$ or $f(0)=-\frac{1}{a^{2}}$. Using the substitutions $x=0$, and $x=0$, $y=-y$ in (12), respectively we get, for all $y \in \mathbb{R}$, the system of equations

$$
\begin{aligned}
f(0) f(y) & =[f(y)]^{2}[f(-y)]^{2} a^{y+4} \\
f(0) f(-y) & =[f(-y)]^{2}[f(y)]^{2} a^{-y+4},
\end{aligned}
$$


then, setting $f(0)= \pm \frac{1}{a^{2}}$, the system

$$
\begin{aligned}
& f(y) a^{-y-6}= \pm[f(y)]^{2}[f(-y)]^{2} \\
& f(-y) a^{y-6}= \pm[f(y)]^{2}[f(-y)]^{2}
\end{aligned}
$$

for $f(y)$ and $f(-y)$.

(13) shows that

$$
f(-y) a^{y-6}=f(y) a^{-y-6} \quad \Leftrightarrow \quad f(-y)=f(y) a^{-2 y} \quad(y \in \mathbb{R}) .
$$

Thus, from the first equation of (13), we get

$$
f(y)\left([f(y)]^{3} a^{-3 y+6} \pm 1\right)=0 \quad(y \in \mathbb{R}) .
$$

Therefore $f(y)=0$ or $f(y)= \pm a^{y-2}$ for $y \in \mathbb{R}$.

Hence, in case $f(0)= \pm \frac{1}{a^{2}}$, the functions

$$
f(x)=\left\{\begin{array}{ll}
a^{x-2} & \text { for } x \in A \\
0 & \text { for } x \in \mathbb{R} \backslash A
\end{array} \quad \text { or } \quad f(x)= \begin{cases}-a^{x-2} & \text { for } x \in A \\
0 & \text { for } x \in \mathbb{R} \backslash A\end{cases}\right.
$$

are the only possible solutions of equation (12) with some sets $A \subseteq \mathbb{R}$.

Similarly as before, we shall prove that the functions in (14) satisfy (12) if, and only if, $A \subseteq \mathbb{R}$ is an arbitrary additive subgroup of $\mathbb{R}$.

Assume that $A \subseteq \mathbb{R}$ is an additive subgroup of $\mathbb{R}$. We show that functions of (14) satisfy (12).

- If $x, y \in A$ then $x+y, x-y \in A$. Thus $f(x)= \pm a^{x-2}, f(y)= \pm a^{y-2}$, $f(x+y)= \pm a^{x+y-2}, f(x-y)= \pm a^{x-y-2}$. Thus a short calculation shows that (12) holds for all $x, y \in A$.

- If $x, y \in \mathbb{R} \backslash A$ then $f(x)=f(y)=0$. Hence, both sides of equation (12) are equal to 0 , which implies that (12) holds in this case, too.

- If $x \in A, y \in \mathbb{R} \backslash A$ then $x+y \in \mathbb{R} \backslash A$. Thus, we infer from $f(x+y)=f(y)=0$ that (12) holds for all $x \in A$ and $y \in \mathbb{R} \backslash A$.

- If $x \in \mathbb{R} \backslash A, y \in A$ then $x-y \in \mathbb{R} \backslash A$. Therefore $f(x)=f(x-y)=0$ immediately implies that $f$ satisfies (12) for all $x \in \mathbb{R} \backslash A$ and $y \in A$.

This completes the proof of our statement.

Now we show that, if the functions of (14) satisfy (12) for some $A \subseteq \mathbb{R}$, then $A$ is an additive subgroup of $\mathbb{R}$ :

- $f(0)= \pm \frac{1}{a^{2}} \neq 0$ implies $0 \in A$; 
- If $y \in A$ then from the first equation of system (14), we get

$$
\pm a^{y-2} a^{-y-6}= \pm a^{2 y-4}[f(-y)]^{2} \quad(y \in A),
$$

which implies that $f(-y) \neq 0$. Thus $-y \in A$;

- If $x, y \in A$ then, replacing $x$ by $x+y$ and $y$ by $x$ in (12), we obtain

$$
f(x+y) f(2 x+y)=[f(x)]^{2}[f(y)]^{2} a^{x+4}=a^{3 x+2 y-4} \neq 0
$$

for all $x, y \in A$. Thus $f(x+y) \neq 0$, that is, $x+y \in A$.

Summarizing, we have proved the following

Theorem 3. The function $f: \mathbb{R} \rightarrow \mathbb{R}$ satisfies functional equation (12), where $1 \neq a \in \mathbb{R}_{+}$is an arbitrary constant if, and only if,

$$
\begin{gathered}
f(x)=0 \text { for } x \in \mathbb{R} \text { or } \\
f(x)=\left\{\begin{array}{ll}
a^{x-2} & \text { for } x \in A \\
0 & \text { for } x \in \mathbb{R} \backslash A
\end{array} \quad \text { or } \quad f(x)= \begin{cases}-a^{x-2} & \text { for } x \in A \\
0 & \text { for } x \in \mathbb{R} \backslash A\end{cases} \right.
\end{gathered}
$$

where $A \subseteq \mathbb{R}$ is an arbitrary additive subgroup of $\mathbb{R}$.

Another solution of Problem 5. Using the addition formula for the exponential function $g(x)=a^{x}(x \in \mathbb{R})$, one can easily obtain that

$$
a^{-x+2} a^{-(x+y)+2}=\left[a^{-y+2}\right]^{2}\left[a^{-(x-y)+2}\right]^{2} a^{-y-4} \quad(x, y \in \mathbb{R}) .
$$

Multiplying equation (12) and (15) side by side, we get the functional equation

$$
f(x) a^{-x+2} f(x+y) a^{-(x+y)+2}=\left[f(y) a^{-y+2}\right]^{2}\left[f(x-y) a^{-(x-y)+2}\right]^{2},
$$

for all $x, y \in \mathbb{R}$, which shows that the function

$$
F(x)=f(x) a^{-x+2} \quad(x \in \mathbb{R})
$$

satisfies (8), that is,

$$
F(x) F(x+y)=[F(y)]^{2}[F(x-y)]^{2} \quad(x, y \in \mathbb{R}) .
$$

Therefore Theorem 2 implies that $F$ satisfies (17) if, and only if, either

$$
F(x)=0 \text { for } x \in \mathbb{R} \text { or }
$$




$$
\text { "meszaros" — 2006/6/22 — 18:17 — page } 189 \text { — \#11 }
$$

$$
F(x)=\left\{\begin{array}{ll}
1 & \text { for } x \in A \\
0 & \text { for } x \in \mathbb{R} \backslash A
\end{array} \quad \text { or } \quad F(x)= \begin{cases}-1 & \text { for } x \in A \\
0 & \text { for } x \in \mathbb{R} \backslash A\end{cases}\right.
$$

where $A \subseteq \mathbb{R}$ is an arbitrary additive subgroup of $\mathbb{R}$. It follows from (16) that $f(x)=F(x) a^{x-2}(x \in \mathbb{R})$. This equality, together with (18), implies Theorem 3.

Remark 7. If $A=\mathbb{R}$ then the functions

$$
f(x)=0 \text { for } x \in \mathbb{R}, \quad f(x)=a^{x-2} \text { for } x \in \mathbb{R}, \quad f(x)=-a^{x-2} \text { for } x \in \mathbb{R}
$$

are the everywhere and nowhere zero solutions of (12), respectively (see [1], [2]).

Remark 8. If $A \subset \mathbb{R}$ is a proper additive subgroup of $\mathbb{R}$, then the not identically zero solutions of (12) are discontinuous at least at the points of $A$, further, if $A=\mathbb{Q}$ then we have a nowhere continuous solution on $\mathbb{R}$.

\section{The generalization of equation (12)}

The given function $g(y)=a^{y+4}(y \in \mathbb{R})$ in equation (12) satisfies the functional equation

$$
g(0) g(x+y)=g(x) g(y) \quad(x, y \in \mathbb{R})
$$

where $g(0)=a^{4}$.

At the second solution of Problem 5, property (19) for the given function $g$ was essential. Further, solution (14) of Problem 5 can be written in the form

$$
f(x)= \pm a^{x-2}= \pm a^{x+4} a^{-6}=g(x)[\sqrt{g(0)}]^{-3} \quad(x \in \mathbb{R}) .
$$

All of these suggest the following generalization of Problem 5.

Problem 6. Find all solutions $f: \mathbb{R} \rightarrow \mathbb{R}$ of the functional equation

$$
f(x) f(x+y)=[f(y)]^{2}[f(x-y)]^{2} g(y) \quad(y \in \mathbb{R})
$$

where the function $g: \mathbb{R} \rightarrow \mathbb{R}_{+}$satisfies equation (19).

By a simple modification of the solutions for Problem 5, we shall prove the following result concerning to the solution of Problem 6 . 
TheOREM 4. Suppose that the function $g: \mathbb{R} \rightarrow \mathbb{R}_{+}$satisfies equation (19). Then the function $f: \mathbb{R} \rightarrow \mathbb{R}$ satisfies equation (20) if, and only if, either

$$
\begin{gathered}
f(x)=0 \text { for } x \in \mathbb{R} \quad \text { or } \quad f(x)=\left\{\begin{array}{ll}
g(x)[\sqrt{g(0)}]^{-3} & \text { for } x \in A \\
0 & \text { for } x \in \mathbb{R} \backslash A
\end{array}\right. \text { or } \\
f(x)= \begin{cases}-g(x)[\sqrt{g(0)}]^{-3} & \text { for } x \in A \\
0 & \text { for } x \in \mathbb{R} \backslash A\end{cases}
\end{gathered}
$$

where $A$ is an arbitrary additive subgroup of $\mathbb{R}$.

Proof. Substitute $x=y=0$ in (20) to get

$$
[f(0)]^{2}=[f(0)]^{4} g(0),
$$

which holds if, and only if, either $f(0)=0$ or $[f(0)]^{2} g(0)=1$.

If $f(0)=0$ then (20), by the substitution $y=0$, implies that $f(x)=0$ $(x \in \mathbb{R})$. that

If $[f(0)]^{2} g(0)=1$ then $f(0) \neq 0, g(0) \neq 0$ and so we obtain from $(20)$ and $(22)$

$$
\frac{f(x)}{f(0)} \frac{f(x+y)}{f(0)}=\left[\frac{f(y)}{f(0)}\right]\left[\frac{f(x-y)}{f(0)}\right]^{2} \frac{g(y)}{g(0)} \quad(x, y \in \mathbb{R}) .
$$

This shows that the functions $F: \mathbb{R} \rightarrow \mathbb{R}, G: \mathbb{R} \rightarrow \mathbb{R}_{+}$, defined by

$$
F(x)=\frac{f(x)}{f(0)}, \quad G(x)=\frac{g(x)}{g(0)},
$$

also satisfy equation (20), that is,

$$
F(x) F(x+y)=[F(y)]^{2}[F(x-y)]^{2} G(y) \quad(x, y \in \mathbb{R})
$$

where $F(0)=G(0)=1$ and $G: \mathbb{R} \rightarrow \mathbb{R}_{+}$satisfies the functional equation

$$
G(x+y)=G(x) G(y) \quad(x, y \in \mathbb{R}) .
$$

Using (25) and $G(0)=1$, one can easily see that $G$ also satisfies the functional equation

$$
G(-x) G(-(x+y))=[G(-y)]^{2}[G(-(x-y))]^{2} G(-y) \quad(x, y \in \mathbb{R}) .
$$


Multiplying (24) and (26) side by side, we get, for all $x, y \in \mathbb{R}$, that

$$
F(x) G(-x) F(x+y) G(-(x+y))=[F(y) G(-y)]^{2}[F(x-y) G(-(x-y))]^{2} .
$$

This implies that the function

$$
H: \mathbb{R} \rightarrow \mathbb{R}, \quad, H(x)=F(x) G(-x)
$$

satisfies equation (8) of Problem 3 and $H(0)=1$. Thus, by Theorem $3, H$ satisfies (8) if, and only if,

$$
H(x)= \begin{cases}1 & \text { for } x \in A \\ 0 & \text { for } x \in \mathbb{R} \backslash A\end{cases}
$$

where $A \subseteq \mathbb{R}$ is an arbitrary additive subgroup of $\mathbb{R}$. Since $f(0)= \pm \frac{1}{\sqrt{g(0)}}$ and $g(x) g(-x)=[g(0)]^{2}$, it follows from $(23),(27)$ and $(28)$ that

$$
f(x)=f(0) g(0) \frac{1}{g(-x)}=\frac{f(0)}{g(0)} g(x)= \pm g(x)[\sqrt{g(0)}]^{-3} \quad(x \in A)
$$

and

$$
f(x)=0 \quad(x \in \mathbb{R} \backslash A) .
$$

This completes the proof of Theorem 4 .

Remark 9. If $g(y)=1(y \in \mathbb{R})$ or $g(y)=a^{y+4}(y \in \mathbb{R})$, we get Problem 4 and Problem 5, respectively.

\section{Final remarks}

These exercises show that the solution of problems containing functional equations opens up a new prospect to us in exercising and studies of different part of curriculum in the secondary education: e.g. solution of equations and system of equations, investigation of special cases in it, taking a survey of the property of operation in the field of the real numbers and its generalizations, counting the value of functions, introducing the continuity of functions, using the method of argue by contradiction.

\section{References}

[1] János Aczél, Vorlesungen über Funktionalgleichungen und ihre Anwendungen, Birkhäuser Verlag, Basel-Stuttgart, 1961. 


$$
\text { "meszaros" — 2006/6/22 — 18:17 — page } 192 \text { — \#14 }
$$

[2] János Aczél, Lectures on functional equations and their application, Mathematics in Science and Engineering vol. 19, Academic Press, New York-London, 1966.

[3] J. Aczél, J. Dhombres, Functional equations in several variables, Encyclopaedia of Math. and its Application, vol. 31, Cambridge University Press, Cambridge-New York-New Rochelle-Melbourne-Sydney, 1989.

[4] Sz. András, L. Kovács, Functional equations, Problem book for students in the 9th and 10th class, (in Hungarian), Ábel Kiadó, Kolozsvár, 2000.

[5] V. Sz. Brodszkij, A. K. Szlipenko, Functional equations in exercises, (in Russian), Visa Skola, Kijev, 1983.

[6] E. Castillo, M. R. Ruiz-Cobo, Functional equations and modelling in science and engineering, Monographs and textbook in applied mathematics, Vol. 161, Marcel Dekker, Inc., New York-Basel-Hong Kong, 1992.

[7] D. Ilse, J. Lehmann, W. Schulz, Gruppoide und Funktionalgleichungen, Mathematik für Lehrer, Band 20 (in Rahmen der Studienbücherei), VEB Deutscher Verlag der Wissenschaften, Berlin, 1984.

[8] A. Járai, Regularity Properties of Functional Equations in Several Variables, Adv. Math. (Dordrecht), Kluwer Acad. Publ., Dordrecht, 2004.

[9] M. Kuczma, Functional equations in a single variable, Vol. 46 of Monographie Mat., P.W.N., Warsaw, 1968.

[10] M. Kuczma, An introduction to the theory of functional equations and inequalities. Cauchy's equation and Jensen's inequality, P.W.N., Warsaw-Krakow-Katowice, 1985.

[11] Károly Lajkó, Functional equations in exercises, lecture notes, (in Hungarian), University of Debrecen, Institute of Mathematics, 2005.

[12] L. Székelyhidi, Convolution type functional equations on topological abelian groups, World Scientific Publishing Co. Inc., Teaneck, NJ, 1991.

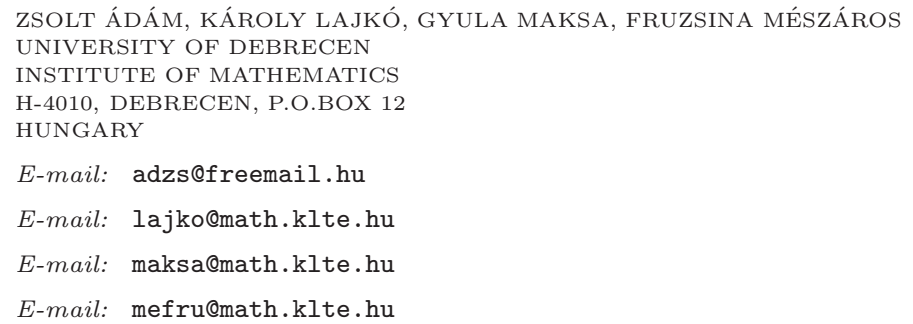

(Received February, 2006) 